\title{
Impact of Using Relationships between Ontologies to Enhance the Ontology Search Results ${ }^{\star}$
}

\author{
Carlo Allocca, Mathieu d'Aquin, and Enrico Motta \\ Knowledge Media Institute (KMi), The Open University, Walton Hall, \\ Milton Keynes, MK7 6AA, United Kingdom \\ $\{$ c.allocca, m.daquin, e.motta\}@open.ac.uk
}

\begin{abstract}
Using semantic web search engines, such as Watson, Swoogle or Sindice, to find ontologies is a complex exploratory activity. It generally requires formulating multiple queries, browsing pages of results, and assessing the returned ontologies against each other to obtain a relevant and adequate subset of ontologies for the intended use. Our hypothesis is that at least some of the difficulties related to searching ontologies stem from the lack of structure in the search results, where ontologies that are implicitly related to each other are presented as disconnected and shown on different result pages. In earlier publications we presented a software framework, KANNEL, which is able to automatically detect and make explicit relationships between ontologies in large ontology repositories. In this paper, we present a study that compares the use of the Watson ontology search engine with an extension, WATSON+KANNEL, which provides information regarding the various relationships occurring between the result ontologies. We evaluate WATSON+KANNEL by demonstrating through various indicators that explicit relationships between ontologies improve users' efficiency in ontology search, thus validating our hypothesis.
\end{abstract}

\section{Introduction}

From the users' perspective, the most important aspect of Semantic Web Search Engines (SWSEs) [7 is the ability to support the search for ontologies which match their requirements. Indeed, finding ontologies is a complex and creative process which requires a lot of intuition. In addition, it also requires manual analyses of the content of candidate ontologies to choose the ones that are adequate to their intended use. For these reasons, the automatic Ontology Selection process has been studied in several different contexts in the recent years

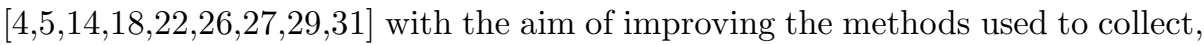
assess and rank candidate ontologies. However, the more user-centric Ontology Search process, here defined as the activity of browsing the results from a SWSE to identify the ontologies adequate to the search goal, has not been researched

\footnotetext{
^ This work was funded by the EC IST-FF6-027595 NeOn Project.
} 
extensively until now. Such an activity is becoming crucial for the rapidly growing set of scenarios and applications relying on the reuse of existing ontologies.

Our view is that one of the issues hampering efficient ontology search is that the results generated by SWSEs, such as Watson (http://watson.kmi.open.ac.uk), Swoogle (http://swoogle.umbc.edu) or Sindice (http://sindice.com), are not structured appropriately. These systems return flat lists of ontologies where ontologies are treated as if they were independent from each other while, in reality, they are implicitly related. For example, the query "Conference Publication" currently 1 gives 218 ontologies as a result in Watson. The first two pages of results list several items, including http://lsdis.cs.uga.edu/projects/semdis/sweto/testbed_v1_1.owl, http://Isdis.cs.uga.edu/projects//semdis/sweto/testbed_v1_3.owl and http://Isdis.cs.uga.edu/projects/semdis/sweto/testbed_v1_4.owl. that represent different versions of the same ontology (isPrevVersionOf). Another common situation is when an ontology has been translated in different ontology languages. This is the case in the first (http://reliant.teknowledge.com/DAML/Mid-level-ontology.owl) and second (http://reliant.teknowledge.com/DAML/Mid-level-ontology.daml) results of the query "student university researcher" in Watson or the second (http://annotation.semanticweb.org/iswc/iswc.daml) and third (http://annotation.semanticweb.org/iswc/iswc.owl) results of the same query in Swoogle. These ontologies are obviously two different encodings of the same model. Analogously, it is not hard to find ontologies connected through other, more sophisticated relations such as different levels of similarity (isLexicallySimilarTo, regarding the vocabulary, and isSyntacticallySimilarTo, regarding the axioms), as well as the relationship between ontologies that originate from the same provenance, as expressed through their URIs having the same second level domain 2 name (ComesFromTheSameDomain) 3

It is our view that the failure of these systems to provide structured views of the result space hamper the ontology search process as the result space becomes unnecessarily large and full of redundancies. Hence, we have been investigating the hypothesis that making explicit the relations between ontologies and using them to structure the results of a SWSE system would support a more efficient ontology search process. In previous publications [1]3, we presented a software framework, KANNEL, which is able to detect and make explicit relationships between ontologies in large ontology repositories. In this paper we present a comparative study evaluating the improvement brought by extending a SWSE (Watson) by making explicit the relationships between ontologies and presenting them in the results of the ontology search task (the WATSON+KANNEL system). To this purpose, we have used a task-oriented and user-centred approach [20.

\footnotetext{
${ }_{1}$ That is, on $16 / 12 / 2011$.

${ }^{2}$ A second-level domain (SLD) is a domain that is directly below a top-level domain such as com, net and org, see http://en.wikipedia.org/wiki/Second-level_domain

${ }^{3}$ In [13] are reported the formal definitions of the above ontology relations.
} 
Based on a sample of users with a suitable profile, we randomly allocated them into two groups and asked them to perform three ontology search tasks to the best of their ability using either the Watson system alone or WATSON+KANNEL. We then evaluated the differences in the way the two groups performed in these tasks, showing through concrete measures as well as responses to questionnaires that the inclusion of relationship between ontologies in the results of a SWSE system improves the efficiency (and to a smaller extent, the satisfaction) of users involved in ontology search tasks.

In the next section, we briefly describe the systems we used in this study. In Section 4, we describe the methodology adopted to evaluate our hypothesis. In Section 5 , we discuss the results of our study. In Section 2, we discuss the relevant related work and in Section [6 we summarise the key contributions of this work and outline our plans for future work.

\section{Related Work}

The discussion of the related work follows two main directions. The first is related to the process of developing Semantic Web Search Engine systems, while the second concerns different types of relationships between ontologies that have been studied in the literature. Most of the research work related to the ontology search task concerns the development of SWSE systems [7, including: Watson [8], Sindice [28], Swoogle [1], OntoSelect [4, ontokhoj [5] and OntoSearch [32]. All these systems have the aim of collecting and indexing ontologies from the web and providing, based on keywords or other inputs, efficient mechanisms to retrieve ontologies and semantic data. To the best of our knowledge, there is no study regarding the comparison of the above ontology search engines. The most common issues addressed by these systems are Ontology Selection - how to identify/select automatically the set of relevant ontologies from a given collection [26 27] - and Ontology Evaluation -how to assess the quality and relevance of an ontology [1427. Several studies have contributed to the solution of both the above problems, including approaches to ranking ontologies [18] and to select appropriate ontologies [22 29:31]. These works focus on the mechanisms required to support SWSE systems in automatically identifying ontologies from their collections and presenting them in a ranked list to the users. However, Ontology Search, as the activity of using a SWSE to find appropriate ontologies, has not been considered before from an user-centric point of view. Furthermore, we can find in literature many works related to the field of Search Engine Usability and how humans interact with search engines [17, but such studies have not yet been applied to SWSEs.

Ontologies are not isolated artefacts: they are, explicitly or implicitly, related to each other. Kleshchev [21] characterised, at a very abstract level, a number of relations between ontologies such as sameConceptualisation, Resemblance, Simplification and Composition, without providing formal definitions for them, and without providing mechanisms to detect them. Heflin [19] was the first 
to study formally some of the different types of links between ontologies, focusing on the crucial problems of versioning and evolution. Although, these links are available with one of the most used web ontology language (OWL), they are rarely used [2]. Several approaches have focused on the comparison of different versions of ontologies in order to find the differences [16. In particular, PROMTDIF 25] compares the structure of ontologies and OWLDiff (http://semanticweb.org/wiki/OWLDiff) computes the differences by entailment, checking the two set of axioms. SemVersion 30. compares two ontologies and computes the differences at both the structural and the semantic levels. Gangemi in [15] defined the ontology integration as the construction of an ontology $\mathrm{C}$ that formally specifies the union of the vocabularies of two other ontologies $\mathrm{A}$ and $\mathrm{B}$. The most interesting case is when $\mathrm{A}$ and $\mathrm{B}$ commit to the conceptualisation of the same domain of interest or of two overlapping domains. In particular, A and B may be related by being alternative ontologies, truly overlapping ontologies, equivalent ontologies with vocabulary mismatches, overlapping ontologies with disjoint domain, or homonymically overlapping ontologies. There also exists an extensive collection of works, including [1012 13 33], that propose formal definitions of the ontology mapping concept. Most of them formalise mappings between concepts, relations and instances of two ontologies, to establish an alignment between them, while we focus on relationships between whole ontologies. Finally, studies have targeted ontology comparison in order to identify overlaps between ontologies 23 and many measures exist to compute particular forms of similarity between ontologies [9].

All these studies discuss particular relations separately. While they contribute interesting elements for us to build on, we focus here on assessing the impact of providing various ontology relations to users of SWSE systems.

\section{Systems Used}

KANNEL is an ontology-based framework for detecting and managing relationships between ontologies for large ontology repositories. Watson is a gateway to the Semantic Web that collects, analyses and gives access to ontologies and semantic data available online. These two systems have already been detailed in 38] respectively. Therefore, in this section we only describe the integration of Watson with KANNEL's features to explain in more details how KANNEL is used on top of Watson's repository and integrated into its interface.

WATSON+KANNEI 4 is an extension of Watson where Watson's ontology space has been processed by KANNEL to detect implicit relationships between ontologies (similarity, inclusion, versioning, common provenance). In addition, two relationship-based mechanisms were added to the Watson ontology search interface (see Fig. 1). They are:

\footnotetext{
${ }^{4}$ The WATSON+KANNEL integration can be tested at http://smartproducts1.kmi.open.ac.uk:8080/WatsonWUI-K
} 
What is it? - Submit URI - Website - Blog - APIs student $\quad$ versions Search Watson

Found 1080 semantic docump

1- http://www.vistology.com/ont/tests/st http://www.vistology.com/ont http://www.vistology.com/ont. http://www.vistology.com/ont.

C http://www.vistology.com/ont 1) http://www.vistology.com/ont

3 similar results 3 other versions:

2- http://www.vistology.com/ont/tests/stu http://www.vistology.com/ont: http://www.vistology.com/ont/tests/student $2.0 w l$ http://www.vistology.com/ont/tests/student 3.owl

C http://www.vistology.com/ont/tests/student4.owl 1) http://www.vistology.com/ont/tests/student4.owl

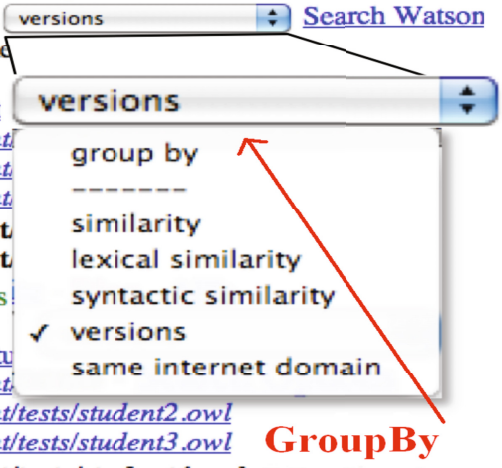

Link $\longrightarrow 3$ similar results 3 other versions 57 results from the same domain

\section{Mechanism}

3- http://www .cs.vu .nl/ kubbe/webkr/model.daml $\$$

C http://www.cs.vu.nV/ kubbe/webkr/model.daml\#Student

P http://www.cs.vu.nl/ kubbe/webkr/model.daml\#has_student

P http://www .cs.vu.nl/ -kubbe/webkr/model.daml\#has_audience

P http://www.cs.vu.nl/ kubbe/webkr/model.daml\#follows_study

C http://www .cs.vu.nl/ kubbe/webkr/model.daml\#Examination_m_'

P http://www .es.vu.nV/ kubbe/webkr/model.daml\#has_course_code

P http://www .cs.vu.nV/ kubbe/webkr/model.daml\#has_to_complete

C http://www.cs.vu.nl/ kubbe/webkr/model.daml\#Studies

C http://www .cs.vu.nV/ kubbe/webkr/model.daml\#Coursecode

P http://www.cs.vu.nV/ kubbe/webkr/model.daml\#has_completed More...

47 results from the same domain

4- http://www.cs.vu.nl/ jkieviet/web/model.daml $\oplus$

C file:/H:/www/web/ideaal.daml\#Student

P file:/H:/www/web/ideaal.daml\#krijgt_les_in

P file:/H:/www/web/ideaal.daml\#krijgt_les_van

P file:/H:/www/web/ideaal.daml\#geeft_les_aan

P file:/H:/www/web/ideaal.daml\#info_uitgever

P file:/H:/www/web/proefje.daml\#nummer

P file:/H:/www/web/ideaal.daml\#letter

P file:/H:/www/web/ideaal.daml\#literatuur

P file:/H:/www/web/ideaal.daml\#is_schrijver_van

P file:/H:/www/web/ideaal.daml\#bepaalt

More...

1 similar results 47 results from the same domain

5- http://www .ontoweb.org/ontology/1

P http://www .ontoweb.org/ontology/1\#student

C http://www .ontoweb.org/ontology/1\#Student

C http://www .ontoweb.org/ontology/1\#PhDStudent

9 similar results

Fig. 1. Watson's interface with KAnnel's features (i.e., WATson+KAnnel) 
Link mechanism which provides additional ontology link 5 to the ontologies in the search result space, depending on the relationships they share with others. For example, three ontology links (3 similar results, 3 other versions and 57 results from the same domain) have been added to the second result in Fig. 1. meaning that there are three ontologies similar to this one in Watson, three other versions and fifty seven that come from the same second level domain.

GroupBy mechanism which provides a feature to support the re-grouping of ontologies in the search result according to a selected ontology relation. For example, in Fig. 1, the search results have been grouped according to the versioning relationship (as it is the one marked as selected), meaning that different versions of the same ontology appear as a single item, represented by the latest version and links to the others. Different levels of similarity (lexical, regarding the vocabulary, and syntactic, regarding the axioms), as well as common provenance can also be used to group results.

\section{Methodology}

The general aim of this study is to evaluate whether the inclusion of ontology relationships to structure the results of a SWSE system, as described in the previous section, improves ontology search. In particular, we consider the following two major aspects to be evaluated:

User efficiency: It is envisaged that ontology relations could benefit users by making the ontology search process more efficient, i.e., requiring less time and effort (as measured by indicators such as the number of pages and ontologies visited).

User satisfaction: It is also hypothesised that, by showing better structured and connected search results, users of the WATSON+KANNEL system would be more satisfied and more confident with the results of their ontology search activity.

The comparative study of Watson and WATSON+KANNEL is based on a taskoriented and user-centred approach [20] that involved participants, tasks and data collections as detailed below.

\subsection{Participants}

Sixteen members of the Open University, from PhD students to senior researchers, participated to the evaluation. They were randomly divided into two groups and asked to perform three ontology search tasks to the best of their ability 6 using

\footnotetext{
${ }^{5}$ The available links are based on the similarity, versioning, common provenance and inclusion relationships as they are described in 113 .

${ }^{6}$ In this work, we considered the tasks to be successfully achieved when the users were satisfied with the ontologies they found.
} 
either Watson or WATSON+KANNEL. We call W the group of 7 participants who used Watson only and $\mathbf{W}+\mathbf{K}$ the group of 9 participants who used WATSON+KANNEL. To make the evaluation unbiased, several requirements had to be fulfilled regarding the ability of users to use the specific systems. The following criteria were used to select participants:

1. Experience with computers and web search engines was necessary.

2. A reasonable degree of understanding of "what an ontology is" and how ontologies might be used in concrete scenarios was needed.

3. A general understanding of what a SWSE is and how it could be used to search ontologies (without necessarily having a direct experience of any of them) was required.

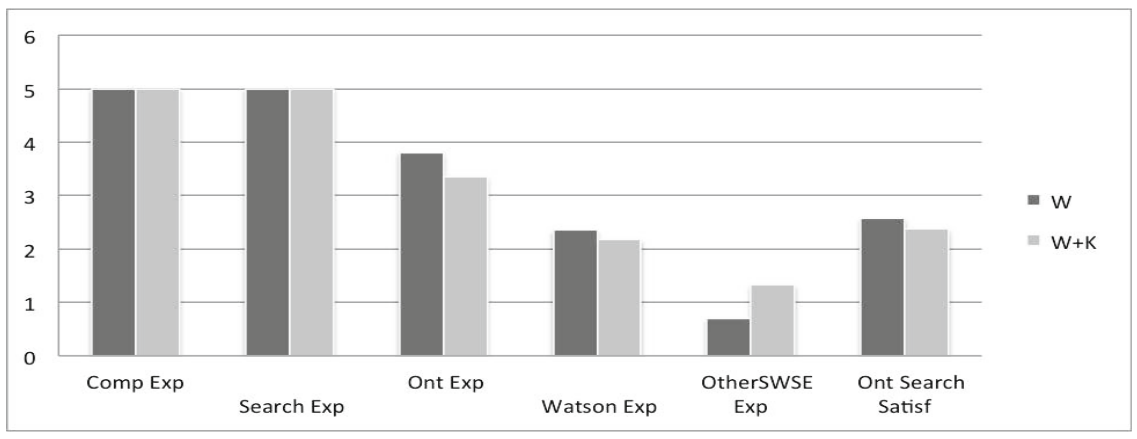

Fig. 2. Profiles of the participants in the two groups $\mathbf{W}$ and $\mathbf{W}+\mathbf{K}$. Answers to the corresponding questions could range from 0 to 5 . The average is shown.

Fig. 2 gives an overview of the profiles of the participants with respect to their experience of different aspects of the evaluation and our three requirements expressed above. It can be seen from the chart that both groups included participants who declared extensive experience of computers in general (Comp Exp) and of Web search engines in particular (Search Exp). In both cases participants had on average a medium to good experience with ontologies (Ont Exp). They generally knew Watson, even if they had not extensively used it before (if at all, see Watson Exp) and some of them had some knowledge of other SWSEs (such as Swoogle and Sindice, see OtherSWSE). When asked about their level of satisfaction with SWSEs, especially in supporting ontology search tasks, participants in both groups gave on average a rather neutral answer (Ont Search Satisf). Moreover, it is worth mentioning that the participants were not aware of the overall goal of the study and were not part of the Watson and WATSON+KANNEL development teams. Thus, regardless of what system they used, they simply performed the ontology search tasks to their best ability, which was actually the only condition needed to ensure the validity of the evaluation. From these results, it appears that participants in our evaluation matched the target audience, as expressed through the requirements above, and formed two homogeneous groups with respect to their experience of the systems involved. 


\subsection{Tasks}

Each individual participant was asked to realise three different ontology search tasks that are described (as presented to the participants) below:

Task 1 - Modelling. You want to develop an ontology about recipes, where you are going to represent the cooking process reusing existing ontologies from the web. Your task is to use Watson (or WATSON+KANNEL) to find ontologies to cover the topic of recipes and processes dealing with cooking.

Task 2 - Annotation. Consider the two links provided7. They are both about the same domain, which is books. Consider them as webpages to which you want to add semantic annotations based on ontologies. To achieve this goal, you need to find ontologies using Watson (or WATSON+KANNEL) that can be used to annotate the above web pages.

Task 3 - Extension. Consider the KMi web page, http://kmi.open.ac.uk/ people/. As you can see, for each person, there is a RDF description represented using the FOAF ontology (http://xmlns.com/foaf/spec/). Imagine that we want to extend the KMi people semantic description including information about the books they have published, the events (conferences, workshops, etc.) they attend and the projects they work on. Your task is to use Watson (or WATSON+KANNEL) to find ontologies that you think would be suitable to be used for such an extension of the existing representation.

\subsection{Data Collection}

The data for evaluation is collected from two main sources: questionnaires and videos. Regarding the first: two main questionnaires were designed for this evaluation. One, regarding the background of users, was filled in by the participants before realising the tasks (cf. participant profiles in Fig. 2). The other one, filled in after realising the tasks, included questions regarding the user's satisfaction and confidence in the results obtained for the three ontology search tasks. Questions in this second questionnaire asked how users felt they succeeded with the tasks, how confident they were about having explored a significant part of the relevant ontology space and their overall opinion about the ability of the tool to support them in the tasks. Videos capturing the screen of participants as they realised the given ontology tasks were used to collect concrete information regarding the performance of users in these tasks. Analysing the videos, we were able to measure the average time taken for each task, the number of pages visited and the number of ontologies inspected.

\footnotetext{
7 http://www . amazon.co.uk/Shockwave-Rider-John-Brunner/dp/0345467175/ref= sr_1_1?ie=UTF8\&qid=1284638728\&sr=1-1-spell and http://www.booksprice.com/compare.do?inputData=top+gear\& Submit2 $\cdot \mathrm{x}=0 \&$ Submit2 $\cdot \mathrm{y}=0 \&$ Submit2=Search\&searchType=theBookName
} 


\section{Results}

The results of our evaluation are presented from both the users' efficiency and satisfaction perspectives. We also discuss how the different ontology relationships included in WATSON+KANNEL were used to support ontology search in the $\mathbf{W}+\mathbf{K}$ group.

\subsection{User Efficiency}

The diagrams in Fig. 3 show the main results of the typical efficiency of the two groups $(\mathbf{W}$ and $\mathbf{W}+\mathbf{K})$ with respect to the three following measures:

Time is the time in minutes taken to realise the task, i.e., between the beginning of the session, until the user was satisfied with the results obtained. This is the most obvious way to assess the performance of users in ontology search.

Page is the number of pages of results an user would have viewed in order to realise a task. This gives an indication of the effort required in browsing the results of the SWSE to identify relevant ontologies.

Link is the number of links followed to realise the task, which corresponds to the number of ontologies being inspected.

It clearly appears in Fig. 3 that our hypothesis (that including ontology relationships in the results of an ontology search system would make the ontology search task more efficient) has been confirmed. Indeed, for the indicator Time and Page, the differences between the $\mathbf{W}$ and $\mathbf{W}+\mathbf{K}$ groups show a significant improvement (taking into account the three tasks, the T-test result for Time was 0.017 and for Page was 0.013, at significance level $\alpha<0.05$ ). While we can observe a difference for the Link indicator, this difference was not shown to be statistically significant (T-test result was 0.27 ). For example, in Task 3, it typically took 2.5 minutes less to achieve the task when using WATSON+KANNEL, and required inspecting only half as many result pages and two third of the links compared to when using Watson only.

It is worth noticing however that there are significant discrepancies in the results obtained for the three different tasks as shown in Fig. 3. In particular, it appears that the differences between $\mathbf{W}$ and $\mathbf{W}+\mathbf{K}$ are less significant in Task 1 . One of the possible explanations for this phenomenon is that it took some time for participants in group $\mathbf{W}+\mathbf{K}$ to explore and learn the features provided by WATSON+KANNEL that were not present in Watson. To support this interpretation, we analysed the videos corresponding to the group $\mathbf{W}+\mathbf{K}$ to determine to what extent the features provided by KANNEL were used in the three ontology search tasks. As shown in Fig. 4, the features of Kannel (especially, the ontology relation links) were used significantly less for Task 1 than they were for Tasks 2 and 3 (see charts A and B). It appears that, after Task 1, users learnt to use the ontology relation mechanisms provided by WATSON+KANNEL more efficiently (see charts C - regarding the Link mechanism - and D - regarding the GroupBy mechanism). 

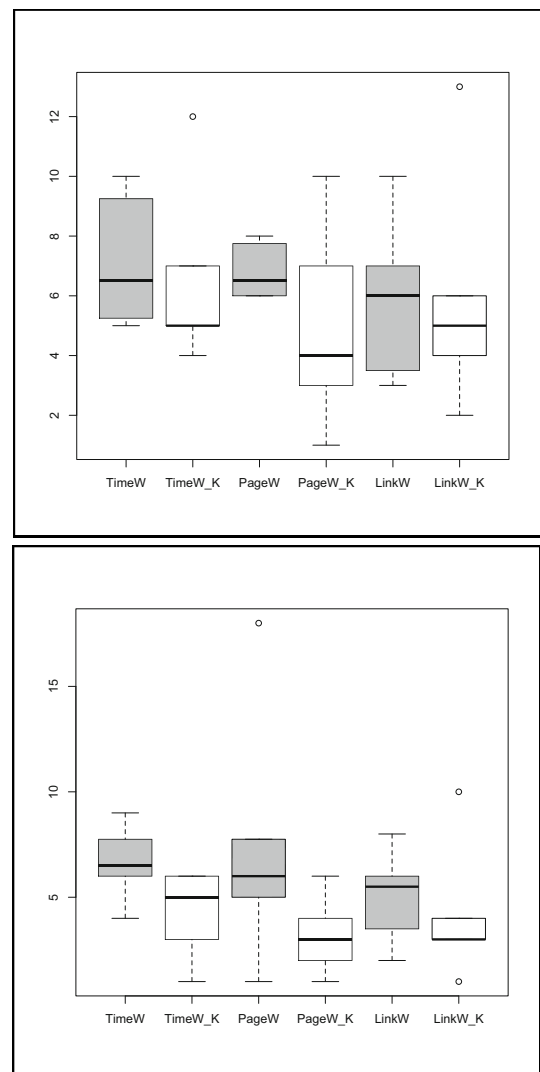

(a) Performance profiles for Task 1.

(b) Performance profiles for Task 2 .

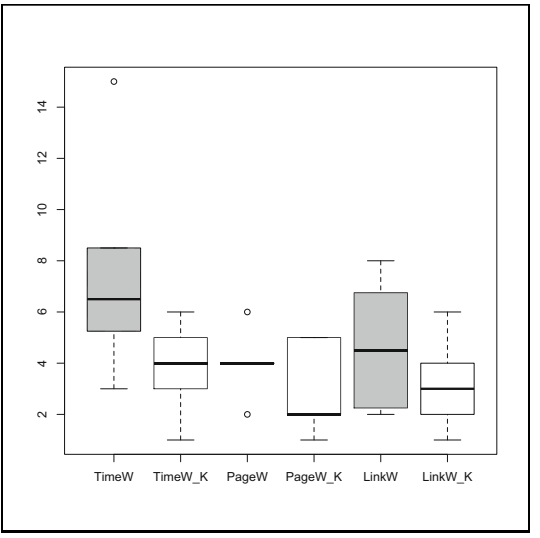

(c) Performance profiles for Task 3.

Fig. 3. Performance profiles for the three ontology search tasks, regarding the Time, Page and Link indicators. Each 'box' represent the median (black line), the quartiles (top and bottom of the box), min and max values for each indicator, in each group for each task. Grey boxes correspond to the profiles of the $\mathbf{W}$ group, white boxes correspond to the $\mathbf{W}+\mathbf{K}$ group. 

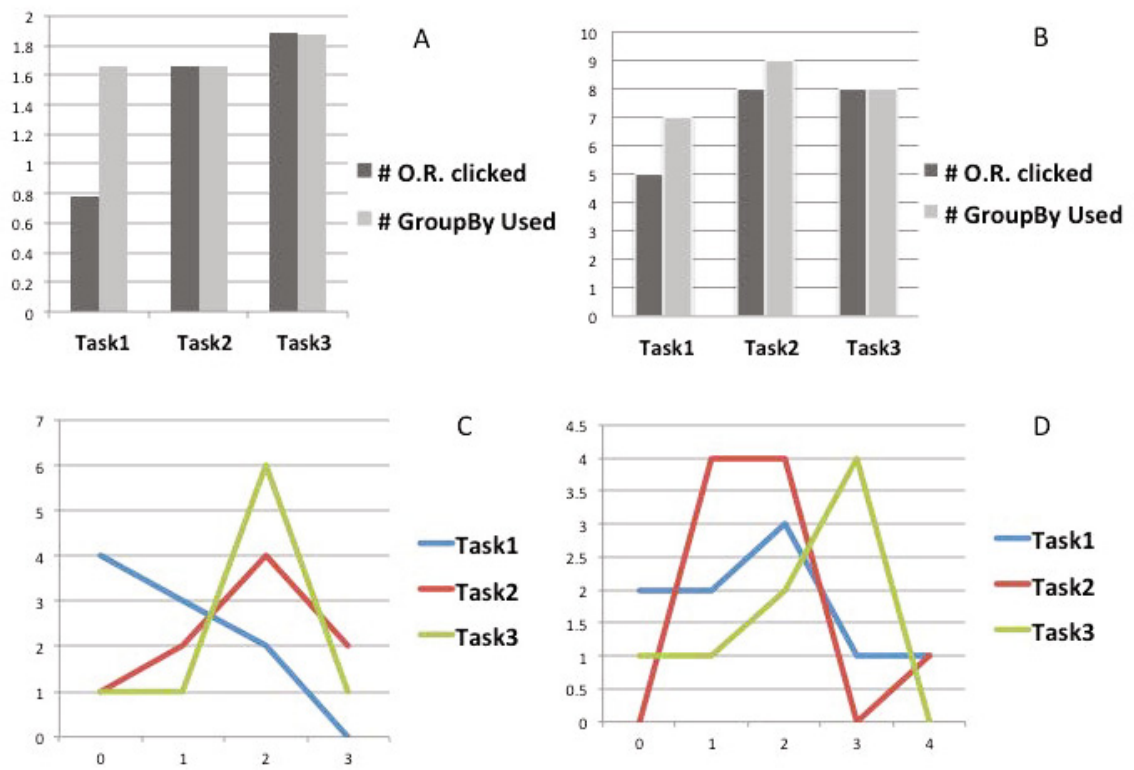

Fig. 4. Use of the ontology relation features in WATSON+KANNEL by group $\mathbf{W}+\mathbf{K}$. (A) shows the average number of ontology relation links followed and the number of times the "group by" mechanism was applied in each task; (B) shows how many participants used these mechanisms in each task. The diagrams (C) and (D) show the distributions of the number of uses (number of times a feature is used - $\mathrm{x}$ axis - by number of users - y axis) for Link and GroupBy respectivly.

\subsection{User Satisfaction}

Fig. 5 summarises the answers to the five questions asked after the users realised the tasks:

1. Are you satisfied with your success with the tasks?

2. Are you satisfied with the ontologies retrieved through Watson?

3. Are you confident that you have seen most of the relevant, retrieved ontologies?

4. Are you satisfied with the search results?

5. Give an indication of your overall opinion regarding your experience of the tested system.

Each question was given an answer from 0 to 5,0 being the most negative and 5 the most positive. Surprisingly, considering that users of the Group $\mathbf{W}+\mathbf{K}$ performed significantly better than the ones of the Group $\mathbf{W}$, there are only very small differences between their answers to most of the user satisfaction questions. Generally, users ranked both systems highly, with the exception of 


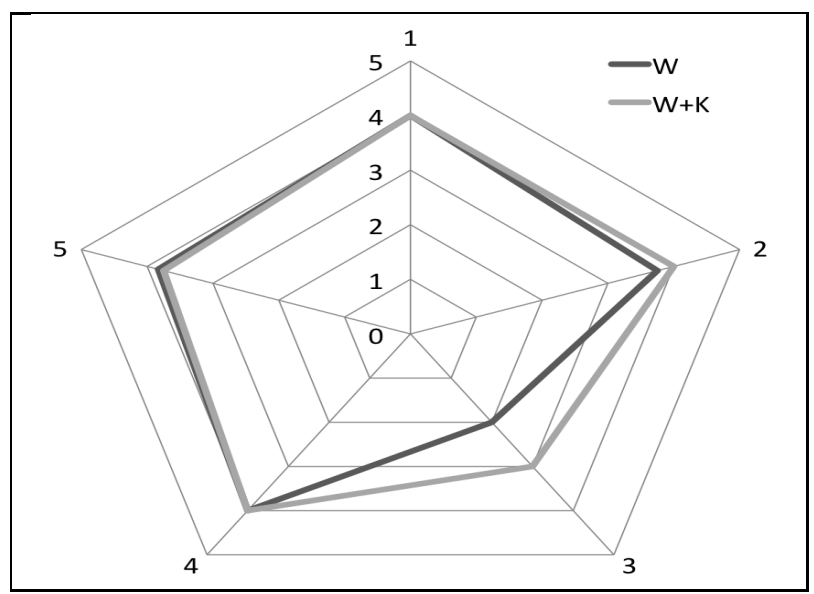

Fig. 5. Median answers to the 5 user satisfaction questions in the two evaluation groups

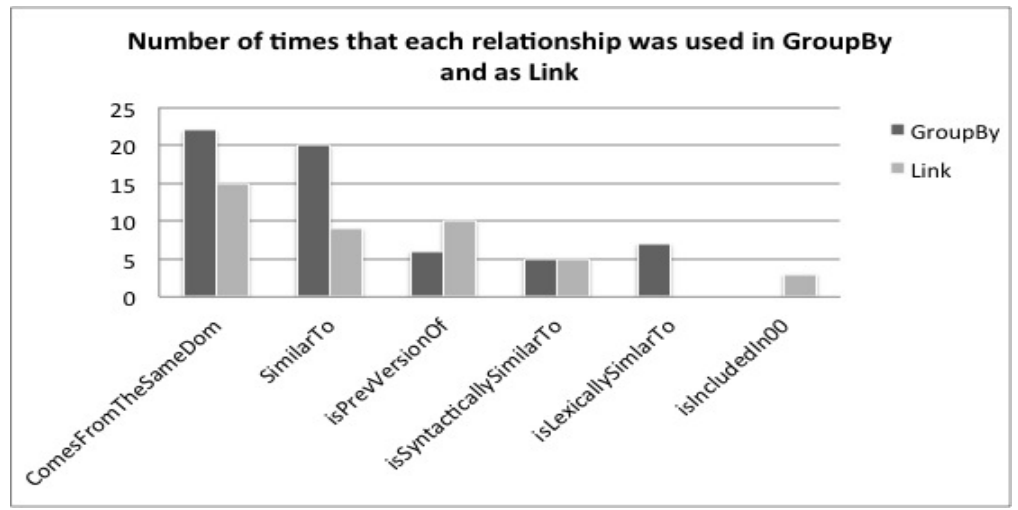

Fig. 6. Use of the 6 relations by the $\mathbf{W}+\mathbf{K}$ group over the three ontology search tasks

Question 3. Question 3 relates to a clear shortcoming of SWSE systems, i.e., the ability to understand and check the whole set of results. This result is consistent with what reported in [24], which shows that expert users tend to rank usability high in systems, regardless of the task performance.

\subsection{Analysis of the Ontology Relationships}

In addition to the users' efficiency and satisfaction, we briefly discuss the extent to which different relations were used to support ontology search. Measures of the use of the Link and GroupBy functions in the WATSON+KANNEL system over the three tasks in group $\mathbf{W}+\mathbf{K}$ are summarised in the charts of Fig. 6 , 
There, it is shown that ComesFromTheSameDomain and SimilarTo (especially in groupBy) are the two most used ontology relationships. One could argue that this is due to the fact that these relations are significantly more present in the Watson repository than any of the others. However, we cannot actually establish a clear correlation between the number of instances of a relationship and its use for the three tasks. Indeed, ComesFrom TheSameDomain is only second in number of relationships (with about four million instances), while SimilarTo is two orders of magnitude more present (more than 100 million instances). More importantly, the third most popular relationship, isPrevVersion, is the least present in the repository with only about 2,800 instances, compared to 14,000 for isIncludedIn (isSyntacticallySimilarTo and isLexicallySimilarTo overlap to a large extent with SimilarTo and with each other, therefore both have more than 100 million instances). A possible explanation for these results is therefore that the three popular relationships (ComesFromTheSameDomain, SimilarTo and isPrevVersion Of ) represent notions that are useful in supporting ontology search. In other terms, being able to discover ontologies that have the same provenance, have a high degree of domain overlap, or represent evolutions of other ontologies appears more natural to users than the use of the isIncludedIn relation. We can also envisage that these three relations are more directly understandable and verifiable than the three others which were less used. Another observation is that relationships with very large numbers of instances (and therefore appearing for many ontologies in the search results) are generally used more with the GroupBy mechanism than with the Link mechanism. This appears natural as 'rare' relationships have less impact then more common ones when used to structure the search results through the GroupBy mechanism.

\section{Conclusion and Future Work}

In this paper, we have presented an evaluation of our hypothesis that, in the context of a Semantic Web Search Engine, making explicit the relationships between ontologies and using them to structure the results of a SWSE system leads to a more efficient ontology search process. This evaluation was based on the comparative study of the Watson search engine and its extension WATSON+KANNEL through a task-oriented and user-centred approach. Both the feedback obtained from questionnaires and concrete performance measures show an improvement in efficiency when performing the ontology search task with WATSON+KANNEL. The current study also provides the basis for several promissing research directions. Firstly, we are interested in extending the evaluation of our hypothesis by conducting a comparative study of other ontology search engines such as Swoogle and Sindice with their KANNEL's extension (SWOOGLE+KANNEL and SINDICE+KANNEL respectively). Secondly, we are interested in building on this work to provide novel ontology ranking solutions. Thirdly, this work also provides the basis for novel empirical studies. In particular, we plan to analyse relationships between ontologies at scale to understand better the ontology engineering practices behind the modelling process. For example, different ontology versions provide data about the development process of ontologies, showing how 
they reach stability or adapt to changes in the domain. Thus, once we have detected the links between different versions of ontologies, it becomes possible to explore how such ontologies evolve on the Semantic Web, in particular with the aim of discovering relevant high level ontology evolution patterns, which can be used to focus ontology search around notions such as 'stability' and 'activity'. Finally, from a practical point of view and as part of our broader work on building a framework for the management of relationships between ontologies (see e.g., [3]), one of our future directions of research is to extend the set of relationships between ontologies that can be considered by our system. One of the most interesting aspects here concerns providing mechanisms to explore not only single, atomic relations between ontologies, but also the relations derived from the combination of others (e.g., compatibility and disagreement [6]).

\section{References}

1. Allocca, C.: Making explic semantic relations between ontologies in large ontology repositories. PhD Symposium at the European Semantic Web Conference (2009)

2. Allocca, C.: Automatic Identification of Ontology Versions Using Machine Learning Techniques. In: Antoniou, G., Grobelnik, M., Simperl, E., Parsia, B., Plexousakis, D., De Leenheer, P., Pan, J. (eds.) ESWC 2011, Part I. LNCS, vol. 6643, pp. 352-366. Springer, Heidelberg (2011)

3. Allocca, C., d'Aquin, M., Motta, E.: Door - towards a formalization of ontology relations. In: Proc. of the Inter. Conf. on Knowledge Engineering and Ontology Development, KEOD, pp. 13-20 (2009)

4. Buitelaar, P., Eigner, T., Declerck, T.: Ontoselect: A dynamic ontology library with support for ontology selection. In: Proceedings of the Demo Session at the International Semantic Web Conference (2004)

5. Chintan, P., Kaustubh, S., Yugyung, L., Park, E.K.: Ontokhoj a semantic web portal for ontology searching, ranking, and classification. In: Proc. 5th ACM Int. Workshop on Web Information and Data Management, New Orleans, Louisiana, USA, pp. 58-61 (2003)

6. d'Aquin, M.: Formally measuring agreement and disagreement in ontologies. In: K-CAP, pp. 145-152. ACM (2009)

7. d'Aquin, M., Ding, L., Motta, E.: Semantic web search engines. In: Domingue, J., Fensel, D., Hendler, J.A. (eds.) Handbook of Semantic Web Technologies, pp. 659-700. Springer, Heidelberg (2011)

8. d'Aquin, M., Motta, E.: Watson, more than a semantic web search engine. Semantic Web Journal 2(1), 55-63 (2011)

9. David, J., Euzenat, J.: Comparison between Ontology Distances (Preliminary Results). In: Sheth, A.P., Staab, S., Dean, M., Paolucci, M., Maynard, D., Finin, T., Thirunarayan, K. (eds.) ISWC 2008. LNCS, vol. 5318, pp. 245-260. Springer, Heidelberg (2008)

10. David, J., Euzenat, J., Šváb-Zamazal, O.: Ontology Similarity in the Alignment Space. In: Patel-Schneider, P.F., Pan, Y., Hitzler, P., Mika, P., Zhang, L., Pan, J.Z., Horrocks, I., Glimm, B. (eds.) ISWC 2010, Part I. LNCS, vol. 6496, pp. 129-144. Springer, Heidelberg (2010) 
11. Ding, L., Finin, T., Joshi, A., Pan, R., Cost, R.S., Peng, Y., Reddivari, P., Doshi, V.C., Sachs, J.: Swoogle: A Search and Metadata Engine for the Semantic Web. In: Proc. of the 13th ACM Conf. on Information and Knowledge Management. ACM Press (November 2004)

12. Ehrig, M.: Ontology Alignment: Bridging the Semantic Gap. Semantic Web and Beyond, vol. 4. Springer, New York (2007)

13. Euzenat, J.: Algebras of Ontology Alignment Relations. In: Sheth, A.P., Staab, S., Dean, M., Paolucci, M., Maynard, D., Finin, T., Thirunarayan, K. (eds.) ISWC 2008. LNCS, vol. 5318, pp. 387-402. Springer, Heidelberg (2008)

14. Gangemi, A., Catenacci, C., Ciaramita, M., Lehmann, J.: A theoretical framework for ontology evaluation and validation. In: Proceedings of SWAP 2005, the 2nd Italian Semantic Web Workshop, Trento, Italy, December 14-16. CEUR Workshop Proceedings (2005)

15. Gangemi, A., Pisanelli, D.M., Steve, G.: An overview of the onions project: Applying ontologies to the integration of medical terminologies. Technical report. ITBM-CNR, V. Marx 15, 00137, Roma, Italy (1999)

16. Gonçalves, R.S., Parsia, B., Sattler, U.: Analysing multiple versions of an ontology: A study of the nci thesaurus. In: Description Logics (2011)

17. Gordon, M., Pathak, P.: Finding information on the World Wide Web: the retrieval effectiveness of search engines. Information Processing \& Management 35(2), 141180 (1999)

18. Alani, H., Brewster, C., Shadbolt, N.: Ranking Ontologies with AKTiveRank. In: Cruz, I., Decker, S., Allemang, D., Preist, C., Schwabe, D., Mika, P., Uschold, M., Aroyo, L.M. (eds.) ISWC 2006. LNCS, vol. 4273, pp. 1-15. Springer, Heidelberg (2006), http://eprints.ecs.soton.ac.uk/12921/01/iswc06-camera-ready.pdf

19. Heflin, J., Pan, Z.: A Model Theoretic Semantics for Ontology Versioning. In: McIlraith, S.A., Plexousakis, D., van Harmelen, F. (eds.) ISWC 2004. LNCS, vol. 3298, pp. 62-76. Springer, Heidelberg (2004)

20. Ingwersen, P.: Information Retrieval Interaction. Taylor Graham (1992)

21. Kleshchev, A., Artemjeva, I.: An analysis of some relations among domain ontologies. Int. Journal on Inf. Theories and Appl. 12, 85-93 (2005)

22. Lozano-Tello, A., Gómez-Pérez, A.: ONTOMETRIC: A Method to Choose the Appropriate Ontology. Journal of Database Management 15(2) (April-June 2004)

23. Maedche, A., Staab, S.: Comparing ontologies-similarity measures and a comparison study. In: Proc. of EKAW 2002 (2002)

24. Motta, E., Mulholland, P., Peroni, S., d'Aquin, M., Gomez-Perez, J.M., Mendez, V., Zablith, F.: A Novel Approach to Visualizing and Navigating Ontologies. In: Aroyo, L., Welty, C., Alani, H., Taylor, J., Bernstein, A., Kagal, L., Noy, N., Blomqvist, E. (eds.) ISWC 2011, Part I. LNCS, vol. 7031, pp. 470-486. Springer, Heidelberg (2011)

25. Noy, N.F., Musen, M.A.: Promptdiff: A fixed-point algorithm for comparing ontology versions. In: 18th National Conf. on Artificial Intelligence, AAAI (2002)

26. Sabou, M., Lopez, V., Motta, E.: Ontology Selection for the Real Semantic Web: How to Cover the Queen's Birthday Dinner? In: Staab, S., Svátek, V. (eds.) EKAW 2006. LNCS (LNAI), vol. 4248, pp. 96-111. Springer, Heidelberg (2006)

27. Sabou, M., Lopez, V., Motta, E., Uren, V.: Ontology selection: Ontology evaluation on the real semantic web. In: 15th International World Wide Web Conference (WWW 2006), Edinburgh, Scotland, May 23-26 (2006) 
28. Tummarello, G., Delbru, R., Oren, E.: Sindice.com: Weaving the Open Linked Data. In: Aberer, K., Choi, K.-S., Noy, N., Allemang, D., Lee, K.-I., Nixon, L.J.B., Golbeck, J., Mika, P., Maynard, D., Mizoguchi, R., Schreiber, G., Cudré-Mauroux, P. (eds.) ASWC 2007 and ISWC 2007. LNCS, vol. 4825, pp. 552-565. Springer, Heidelberg (2007), http://dblp.uni-trier.de/db/conf/semweb/iswc2007.html\#TummarelloD007

29. Hong, T.-P., Chang, W.-C., Lin, J.-H.: A Two-Phased Ontology Selection Approach for Semantic Web. In: Khosla, R., Howlett, R.J., Jain, L.C. (eds.) KES 2005. LNCS (LNAI), vol. 3684, pp. 403-409. Springer, Heidelberg (2005)

30. Volkel, M.: D2.3.3.v2 SemVersion Versioning RDF and Ontologies. EU-IST Network of Excellence (NoE) IST-2004-507482 KWEB

31. Xiaodong, W., Guo, L., Fang, J.: Automated ontology selection based on description logic. In: CSCWD, pp. 482-487 (2008)

32. Zhang, Y., Vasconcelos, W., Sleeman, D.H.: Ontosearch: An ontology search engine. In: Bramer, M., Coenen, F., Allen, T. (eds.) SGAI Conf., pp. 58-69. Springer, Heidelberg (2004)

33. Zimmermann, A., Krötzsch, M., Euzenat, J., Hitzler, P.: Formalizing ontology alignment and its operations with category theory. In: Proceeding of the 4th Inter. Conf. on Formal Ontology in Information Systems, FOIS, pp. 277-288. IOS Press (2006) 